\title{
Toxoplasmosis Options Now and in the Future
}

\section{Abdulaziz Radhi S Aljohni ${ }^{1 *}$, Ahad Amer Alsaiari ${ }^{2}$, Omar Abdulrhman Alfaroq ${ }^{3}$, Azeza Abdulrhman Alfaroqie ${ }^{3}$, Abdulrahman Alhomadi Alanezi ${ }^{4}$, Majed Saad Alahmadi ${ }^{5}$, Tariq Ibrahim Alhazmy ${ }^{6}$, Ameenah Mualla Saad Alharbi ${ }^{7}$ and Bandar Mulfi Alluqmani ${ }^{8}$}

${ }^{1}$ Phd Microbiology, Department of Laboratory at King Fahad Hospital, Saudi Arabia

${ }^{2}$ College of Applied Medical Science, Department of Clinical Laboratory Sciences, Taif University, Saudi Arabia

${ }^{3}$ Department of Laboratory at King Fahad Hospital, Medina, Saudi Arabia

${ }^{4}$ Chief Technologist of Laboratory, King Fahad Hospital, Medina, Saudi Arabia

${ }^{5}$ MSc in Biomedical Science (Blood Science) at Madina Regional Laboratory and

Central Blood Bank, Saudi Arabia

${ }^{6}$ MSc Laboratory Histopathology at Al-Qunfudhah Health Regional Laboratory,

Saudi Arabia

${ }^{7}$ Nursing Specialist, North Makkah Health Sector, Saudi Arabia

${ }^{8}$ Laboratory Technician Microbiology, King Abdulaziz Hospital, Saudi Arabia

*Corresponding Author: Abdulaziz Radhi S Aljohni, Phd Microbiology, Department of Laboratory at King Fahad Hospital, Saudi Arabia.
Received: January 13, 2022

Published: February 11, 2022

(C) All rights are reserved by Abdulaziz Radhi

S Aljohni., et al.

\begin{abstract}
Toxoplasmosis is a zoonotic disease caused by the protozoan parasite Toxoplasma gondii, which is transmitted from person to person. Even though felids are the most common hosts of $T$. gondii, encysted parasites can survive in the tissues of almost any host for an extended period, possibly even for the rest of their lives. Certain clinical cases are the consequence of fresh T. gondii exposures, whereas others are the result of parasites in tissue cysts becoming reactivated, as has happened in some cases recently. Among warm-blooded species, including humans, T. gondii infection is frequent. In immunocompetent, non-pregnant persons who are not pregnant, infection usually results in no sickness or just moderate clinical signs. In contrast, infections acquired during pregnancy can result in minor to severe congenital abnormalities in the fetus, and immunocompromised humans or animals can have severe, life-threatening diseases as a result of these infections. Serious and life-threatening infections in immunocompetent adults in French Guiana and Suriname have recently raised the prospect that some extremely virulent strains of Toxoplasma gondii may occur in tropical woods, according to new findings.
\end{abstract}

Keywords: Toxoplasmosis; Protozoan Parasite; Toxoplasma gondii; Pathogenesis; History; Cats and Toxoplasmosis; Effect on Pregnancy

\section{Introduction}

An apicomplexan parasite, Toxoplasma gondii infects humans and most other warm-blooded animals [1]. When it comes to pregnancy or immunosuppressive diseases and treatments, T. gon- dii infection can lead to sickness and death in healthy individuals $[2,3]$. Eleven chromosomes make up the $80 \mathrm{Mb}$ genome [4]. When it comes to the related malarial pathogen Plasmodium falciparum, T. gondii serves as an excellent model system $[5,6]$. We may learn a 
great deal from EST [7] and unfinished draft sequences, as revealed by the results of this study [8]. Research in the disciplines of malaria and apicomplexan biology has been considerably facilitated by the availability of draft sequences, databases, and data mining techniques. Most eukaryotic genome projects, especially those in non-model organisms, may never be completed despite the completion of the genome of P. falciparum. The T. gondii genome will be sequenced using a random shotgun technique with 3-10X coverage on a large number of genomes. Most organism-specific technologies, such as gene discovery algorithms, are still in the development or training stages, for better or for worse. When dealing with draft sequences, the goal is to design tools and tactics that will help optimize the usefulness and avoid the difficulties that come with interpreting unfinished datasets, which are becoming increasingly widespread (redundancy in the data, sequencing, and assembly errors, contaminating sequences, etc.).

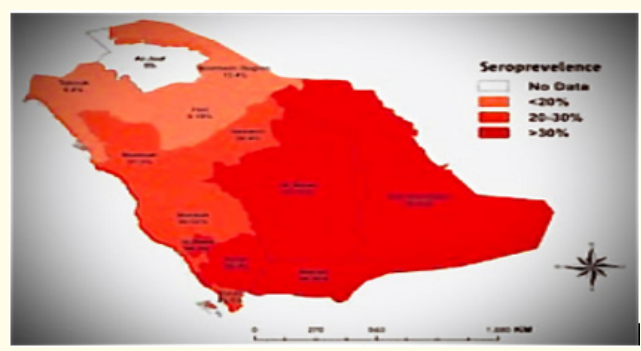

Figure 1: In Saudi Arabia, the incidence of chronic toxoplasmosis varies according to the administrative area.

\section{Pathogenesis}

Food contaminated with cat feces containing oocysts or infected meat having tissue cysts harboring bradyzoites are the most common sources of toxoplasmosis in humans, with the vast majority of cases occurring as a result of consuming infected meat. Brachyzoites and sporozoites infect intestinal cells and disseminate locally to mesenteric lymph nodes before spreading to distant organs through the lymphatic system and the bloodstream. Necrosis can develop in a range of organs, and the clinical signs of the condition reflect the extent of the tissue damage. Tissue death is not induced by a Toxoplasma toxin, but rather by the expulsion of tachyzoites from the host cell, which causes the host cell to die. When toxoplasma infections are symptomatic, only 10-30 percent of cases are reported, with lymphadenitis and lymphadenopathy being the most prevalent clinical symptoms in immunocompetent people. Swollen lymph nodes are the most prevalent symptom, which may be accompanied by other symptoms such as fever, headache, muscle pain, anemia, and, in rare cases, lung issues. Fever, headache, muscle discomfort, anemia, and lung complications are also possible. Even though any lymph node can get infected, those located deep in the neck are those most frequently afflicted. It is characterized by symptoms such as malaise, fever, and lymphocytosis that are indicative of infectious mononucleosis. When an illness persists for many weeks or months, it is typical for it to disappear by itself.

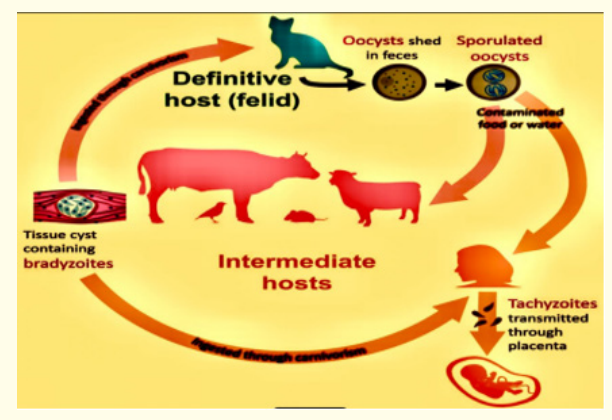

Figure 2: Toxoplasma gondii's life cycle is shown in the diagram. Bradyzoites play a crucial role in the transmission of parasites, whether between intermediate hosts or to the final hosts in the parasite's life cycle.

\section{Taxonomy}

Toxoplasma gondii is classified as part of the Apicomplexa phylum (Levine 1970), the Sporozoasida class (Leuckart 1879), and the Coccidiasina subclass (Leuckart 1879). (Leukart 1879). Until 1970, all coccidia were classed as members of the family Eimeriidae. Following the 1970 discovery of $T$. gondii's coccidian cycle, $T$. gondii has been classified by several sources as belonging to the families Eimeriidae, Sarcocystidae, or Toxoplasmatidae.

\section{History}

They found it in a North African rat, Ctenodactylus gondii, in 1908. The gondi rodent is the host of Toxoplasma gondii (toxin, arc, or bow as in bow and arrow, Greek, and plasma, form, Greek). T. gondii was discovered in Panama in a lab rabbit and then in a person. In 1909, Chatton and LeBlanc recommended that gondis from Tunisia's slopes and highlands be infected in the lab. The parasite was identified in the gondi's blood, spleen, and liver at the Pasteur 
Institute in Tunis. Several researchers in Tunis and the US failed at arthropod transmission. A hydrocephalic child's retina and congenital toxoplasmosis were identified in 1923, prompting interest in human illnesses. For toxoplasmosis, Albert Sabin (of the polio vaccine fame) developed a particular serological "dye test" with Henry Feldman in under 5 years. The dye test identified toxoplasmosis as a human infection with global distribution. Congenital infections could not account for their broad incidence for over 35 years. When subjected to proteolytic enzymes, cysts disintegrated, releasing viable infective stages, as proposed by D. Weinman and Asa Chandler in 1956. Undercooked lamb chops or barely cooked beef or horse meat were served to children in a Paris TB hospital in Desmonts., et al. 1965's studies. Antibody levels (measured by the Sabin-Feldman dye test) jumped from $10 \%$ to $100 \%$ in the former group, according to the results. Toxoplasmosis was found in over $80 \%$ of people in Paris, where raw or undercooked meat is regularly eaten. A puzzling question remained: why is toxoplasmosis prevalent in both meat-eaters and non-meat eaters, i.e., vegetarians and herbivores? Tachyzoites and bradyzoites were the only two phases known up until 1970, when a third step was discovered: a third stage known as a syncytium. Apical complexes were found in the invasive stages of T. gondii, suggesting it was an apicomplexan. In 1970, oocysts and sexual phases were discovered in cat intestine cells, completing the life cycle.

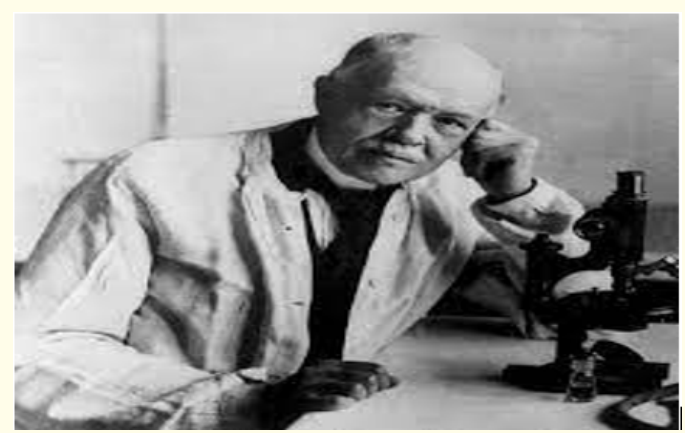

Figure 3: Charles Nicolle (1866-1936) | Institut Pasteurpasteur.fr.

\section{Risk factors}

Anyone may get infected with T. gondii at any time. For pregnant women and persons with compromised immune systems, toxoplasmosis is particularly harmful, since they are more susceptible to infection. Patients suffering from AIDS, those who have had organ transplants, and those on immunosuppressive medicines are all examples of this.

\section{The life cycle of toxoplasmosis is as follows}

Oocysts sporulate and become pathogenic in the environment after 1-5 days. In nature, intermediate hosts (such as birds and rodents) get infected by consuming oocyst-contaminated soil, water, or plant material. Tachyzoites develop quickly after ingesting oocysts.

\section{Cats and toxoplasmosis}

Toxoplasma gondii causes toxoplasmosis. Humans, children, and other animals may also be infected. Infection occurs when humans or animals consume the parasite's microscopic eggs or cysts in raw meat from cattle, sheep, pigs, or wild animals like deer.

T. gondii can only mature in a cat's body. The adult parasite lives in cats' stomachs, and the eggs are spread by their feces. The eggs must mature in the soil for 15- days before they may infect people or other animals. It hatches within the colon and burrows through the intestinal wall when eaten by humans or other animals. When the parasite penetrates a person (an unfavorable host), it creates a cyst in a muscle or organ. Later in life, the cysts may recur, particularly if the immune system is compromised by sickness or medication.

\section{Pregnancy complications}

Except for women who are infected with T. gondii up to 3 months before conception, infection with T. gondii before pregnancy is expected to cause little or no damage to the unborn child, according to current research. A neonate with hydrocephalus, microcephaly, intracranial calcifications, retinochoroiditis, strabismus, blindness, epilepsy, psychomotor and mental retardation, petechiae due to thrombocytopenia, and anemia may have congenital toxoplasmosis, according to the Centers for Disease Control and Prevention. The probability of fetal transfer is modest in the first trimester (less than 6 percent), but it is high in the third trimester (between 60 and 80\%). T. gondii infection during embryogenesis, on the other hand, albeit rare, has far-reaching implications for the baby [12]. Maternal sickness during the third trimester, on the other hand, 
commonly results in neonates who are asymptomatic at birth. However, if these newborns do not receive the correct treatment, they may develop retinochoroiditis and neurologic deficits in adolescence or early adulthood, which can be fatal. Breastfeeding or direct human-to-human contact has not been shown to transmit $T$. gondii. [11,13].

\section{Toxoplasmosis pediatric}

Toxoplasmosis is a disease caused by infection with the Toxoplasma gondii parasite, which is a parasite that may be found in many parts of the world and is rather common. A parasite infection can have serious effects in patients with weakened immune systems or in children born to mothers who have been infected with the parasite. Most pregnancies end in miscarriage for women who become infected with the parasite during the first trimester of their pregnancy.

\section{Symptoms of toxoplasmosis}

The majority of healthy individuals afflicted with toxoplasmosis exhibit no symptoms and are unaware they are sick. The flu is characterized by bodily aches, enlarged lymph nodes, and fever. a migraine, a Fatigue Several of the most severe symptoms seen in immunocompromised (AIDS patients) or immunosuppressed (cancer, organ transplant) persons include the following, Symptoms of Pneumocystis jiroveci pneumonia, a common opportunistic infection seen in AIDS patients. Blurred vision is caused by retinal inflammation (ocular toxoplasmosis). A mother's infection during the third trimester puts her infant at risk for toxoplasmosis, but infection during the first 14 trimesters does not. The earlier in pregnancy an infection develops, the more disastrous the outcome for the kid. Numerous infants who are infected at an early age die. Survivors are predisposed to serious difficulties such as the following. The liver and spleen have grown in size. Yellowing of the skin and eyes (jaundice). Infected eyes.

\section{Diagnosis}

Retinochoroiditis is diagnosed by an ophthalmologist. AntiToxoplasma treatment is indicated if typical lesions (white localized lesions typically accompanied by vitreous inflammatory responses) occur together with Toxoplasma seropositivity. Biological tests are essential for individuals with uncommon ocular lesions or who do not respond to anti-Toxoplasma medication. Both parasite detection and local antibody production in ocular fluids are complementary processes. It has been claimed that quantitative PCR techniques may identify parasite DNA in AH or vitreous fluid, with sensitivity ranging from 16 to 55 percent [14]. There is some evidence that Toxoplasma DNA is more likely to be found in immunocompromised patients' aqueous humor, but the data is restricted and prospective data are lacking. In contrast to Westeneng., et al. Talabani., et al. [15] found parasite DNA in 8/11 patients (73 percent), with PCR being the only positive test in 4 cases. In a group of patients with atypical retinochoroiditis, PCR failed to detect 9 individuals who responded to anti-Toxoplasma treatment, according to Favreau., et al.

Antibody detection in ocular fluids is a useful adjuvant in the diagnosis of ocular toxoplasmosis. Antibody production in an ocular fluid may be measured or approximated subjectively by comparing serum IgG titers. The Goldmann-Witmer coefficient (GWC) may detect aberrant levels of anti-Toxoplasma antibodies in aqueous humor [56]. + [serum total IgG (g/liter)/AH total IgG (g/liter)]. Turunen., et al. proposed comparing anti-Toxoplasma antibody titers in AH to mumps virus antibody titers [16]. In one study, repeated sampling at weekly intervals boosted the GWC's sensitivity from $57 \%$ to $70 \%$. However, late sampling has been cited for poor PCR findings in certain articles. The duration between the start of symptoms and paracentesis seems to be significant for identifying certain band patterns in serum and matched AH samples by Western blot analysis, with longer intervals producing better results. The WB's sensitivity is similar to the GWC's (53-81\%) $[15,16]$, but its specificity is higher ( $>95 \%$ ) due to reduced inflammation and blood-retina barrier disruption.

The detection of specific IgA in $\mathrm{AH}$ ac has also been shown to be useful, either as a quantitative indicator of local antibody production by comparing serum and $\mathrm{AH}$ titers or as a qualitative marker by comparing WB patterns, with sensitivities of 63 percent and 76 percent, respectively, for both quantitative and qualitative purposes.

Using GWC, PCR, and WB together boosts biological efficiency. diagnostic sensitivity, reaching $83-85 \%$ when data from numerous large case series are merged [17]. Based on the patient's characteristics and the time of $\mathrm{AH}$ puncture, several writers established the following algorithm: In the first 10 days after symptoms start, real- 
time PCR should be used first, especially if the patient is immunocompromised or if the total size of the foci is large ( $>2$ optic disc diameters); (ii) use of GWC after the first 10 days, if old scars are present and/or the anterior chamber reaction, is mild to severe, associated with PCR; Labalette., et al. found that combining AH antibody load measurements with PCR boosts diagnostic sensitivity independent of time to diagnosis or amount of lesions. For lesions larger than three disk areas, PCR alone is more sensitive [18].

\section{Prevalence}

According to estimates, between 25 and 30 percent of the world's population is infected with Toxoplasma gondii. [19].

In the United States, 89 percent of women of reproductive age are at risk of acute infection and of transferring the parasite to the infant if the initial infection occurs during the gestational period [20].

The age-adjusted seroprevalence rate in the United States is 22.5 percent. The distribution varies significantly, with the greatest incidence recorded in the northeastern regions and the lowest in the western states [21].

Low seroprevalences of $10 \%$ to $30 \%$ have been found in places such as North America, Northern Europe, and the Sahelian regions of Africa. Seroprevalence ranges from 30 to 50 percent in Central and Southern European nations, tropical African countries, and Latin American countries. This demonstrates the variance within nations as well as across countries [22].

\section{Incidence}

- $\quad$ Toxoplasmosis affects 1.1 million persons in the United States each year [23].

- Every year, 500 to 4000 newborns are affected with congenital toxoplasmosis [24].

\section{Conclusion}

In the past 20 years, Toxoplasma research has advanced significantly. High-throughput genomics and proteomics, as well as innovative imaging approaches, assisted fundamental molecular and cell biology research. In order to describe these enhancements, further study is required. In the meanwhile, epidemiological investigations have gained popularity. Since the development of Toxoplasma as a waterborne illness, researchers have focused their attention on the environment. An ecological and integrated strategy was developed to better understand Toxoplasma's complex hostenvironment interactions and risk factors for human infection. In order to discover the genetic makeup of many different animal species from throughout the globe, they were separated and genotyped. T. gondii's variation. We now know that this unique species does not exist anywhere on the earth. The pathogenic impact of several of these genotypes has been extensively studied in vitro. These models helped find virulence genes. Toxoplasma genotypes and geographic distribution are being studied in order to better understand the clinical manifestations of toxoplasmosis, it seems. To treat human toxoplasmosis effectively, these new epidemiological and genetic discoveries must be validated. However, finding new indicators for the most difficult instances, such as ocular or cerebral localizations, should be advantageous.

\section{Bibliography}

1. Dubey JP. "Toxoplasma, Hammondia, Besnoita, Sarcocystis and other tissue cyst forming coccidia of man and animals". In Kreier, J.P. (ed.), Parasitic Protozoa III. Academic Press, New York, NY (1997): 101-237.

2. Remington JS and Desmonts G. "Toxoplasmosis". In Remington, J.S. and Klein, J.O. (eds), Infectious Diseases of the Fetus and Newborn Infant. W. B. Saunders, Philadelphia, PA (1989): $89-195$

3. Luft BJ and Remington JS. "Toxoplasmic encephalitis in AIDS patients". Clinical Infectious Diseases 15 (1992): 211 -222.

4. Sibley LD and Boothroyd JC. "Construction of a molecular karyotype for Toxoplasma gondii". Molecular and Biochemical Parasitology 51 (1992): 291-300.

5. Ajioka JW. "Analysis of apicomplexan parasites". Methods 13 (1997): $79-80$.

6. Roos DS., et al. "Toxoplasma as a model parasite: apicomplexan biochemistry, cell biology, molecular genetics... and beyond". In Tschudi, C. and Pearce, E. (eds), Biology of Parasitism. Kluwer Press, Boston, MA (1999): 143 -167. 
7. Ajioka JW., et al. "Gene discovery by EST sequencing in Toxoplasma gondii reveals sequences restricted to the Apicomplexa”. Genome Research 8 (1998): 18-28.

8. Janssen CS., et al. "Gene discovery in Plasmodium chabaudi by genome survey sequencing". Molecular and Biochemical Parasitology 113 (2001) 251-260.

9. Gavinet MF., et al. "Congenital toxoplasmosis due to maternal reinfection during pregnancy". Journal of Clinical Microbiology 35.5 (1997): 1276-1277.

10. McAuley J., et al. "Early and longitudinal evaluations of treated infants and children and untreated historical patients with congenital toxoplasmosis: the Chicago Collaborative Treatment Trial". Clinical Infectious Diseases 18.1 (1994): 38-72.

11. Montoya JG and Liesenfeld O. "Toxoplasmosis". Lancet 363.9425 (2004): 1965-1976.

12. Dunn D., et al. "Mother-to-child transmission of toxoplasmosis: risk estimates for clinical counselling". Lancet 353.9167 (1997): 1829-1833.

13. Berrébi A., et al. "Long-term outcome of children with congenital toxoplasmosis". American Journal of Obstetrics and Gynecology 203.6 (2010): 552.e1-6.

14. Fekkar A., et al. "Comparison of immunoblotting, calculation of the Goldmann-Witmer coefficient, and real-time PCR using aqueous humor samples for diagnosis of ocular toxoplasmosis". Journal of Clinical Microbiology 46 (2008): 1965-1967.

15. Talabani H., et al. "Contributions of immunoblotting, real-time PCR, and the Goldmann-Witmer coefficient to diagnosis of atypical toxoplasmic retinochoroiditis". Journal of Clinical Microbiology 47 (2009): 2131-2135.

16. Turunen HJ., et al. "Demonstration of intraocular synthesis of immunoglobulin G Toxoplasma antibodies for specific diagnosis of toxoplasmic chorioretinitis by enzyme immunoassay". Journal of Clinical Microbiology 17 (1983): 988-992.
17. Villard 0., et al. "Comparison of enzyme-linked immunosorbent assay, immunoblotting, and PCR for diagnosis of toxoplasmic chorioretinitis". Journal of Clinical Microbiology 41 (2003): 3537-3541.

18. Labalette P., et al. "Ocular toxoplasmosis after the fifth decade". American Journal of Ophthalmology 133 (2002): 506-515.

19. Montoya JG and Liesenfeld O. "Toxoplasmosis". Lancet 363.9425 (2004): 1965-1976.

20. Montoya JG and Remington JS. "Management of Toxoplasma gondii infection during pregnancy". Clinical Infectious Diseases 47.4 (2008): 554-566.

21. Jones JL., et al. "Toxoplasma gondii infection in the United States: seroprevalence and risk factors". American Journal of Epidemiology 154.4 (2001): 357-365.

22. Pappas G., et al. "Toxoplasmosis snapshots: global status of Toxoplasma gondii seroprevalence and implications for pregnancy and congenital toxoplasmosis". International Journal for Parasitology 39.12 (2009): 1385-1394.

23. CDC - Toxoplasmosis - Epidemiology and Risk Factors.

24. Lopez A., et al. "Preventing congenital toxoplasmosis". $M M W R$ Recommendations and Reports 49 (2000): 59-68.

\section{Assets from publication with us}

- Prompt Acknowledgement after receiving the article

- Thorough Double blinded peer review

- Rapid Publication

- Issue of Publication Certificate

- High visibility of your Published work

Website: www.actascientific.com/

Submit Article: www.actascientific.com/submission.php

Email us: editor@actascientific.com

Contact us: +919182824667 